\title{
Procedimentos empregados na estimativa das medidas de massa e de volume de alimentos selecionados pelo Inquérito Nacional de Alimentação 2008-2009
}

\author{
Procedures for estimating the weight and volume \\ of selected foods reported in cooking units by \\ the National Dietary Survey of 2008-2009
}

\author{
Ilana Nogueira BEZERRA ${ }^{1}$ \\ Luana Silva MONTEIRO2 \\ Marina Campos ARAUJO ${ }^{1}$ \\ Rosangela Alves PEREIRA ${ }^{2}$ \\ Edna Massae YOKOO3 \\ Rosely SICHIERI ${ }^{1}$
}

RE S U M O

\section{Objetivo}

Descrever os procedimentos utilizados para estimar as medidas de massa e de volume dos itens mais consumidos no Brasil e apresentar propostas que possam ser adotadas para o aprimoramento do cálculo das quantidades ingeridas nos inquéritos alimentares no Brasil.

\section{Métodos}

Foram utilizados os dados do Inquérito Nacional de Alimentação 2008-2009 para descrever as frequências brutas das unidades de medida referidas para os cinco itens mais consumidos no Brasil (arroz, feijão, café, pão e carne bovina frita), exemplificou-se a construção da base de dados utilizada no Inquérito Nacional de Alimentação e discutiram-se as principais dificuldades encontradas nesse processo.

\section{Resultados}

Foram citadas aproximadamente 11800 unidades de medidas associadas a cerca de 2000 alimentos/ preparações. A colher de servir foi a medida mais citada para o arroz (57\%); a concha, para o feijão (80\%); a

\footnotetext{
${ }^{1}$ Universidade do Estado do Rio de Janeiro, Instituto de Medicina Social, Departamento de Epidemiologia. R. São Francisco Xavier, 524, $7^{\circ}$ andar, sala E7002, Maracanã, 20550-900, Rio de Janeiro, RJ, Brasil. Correspondência para/Correspondence to: R SICHIERI. E-mail: <sichieri@ims.uerj.br>.

2 Universidade do Estado do Rio de Janeiro, Instituto de Nutrição Josué de Castro, Departamento de Nutrição Social Aplicada. Rio de Janeiro, RJ, Brasil.

${ }^{3}$ Universidade Federal Fluminense, Centro de Ciências Médicas, Departamento de Epidemiologia e Bioestatística. Niterói, RJ, Brasil.
} 
646 | IN BEZERRA et al.

xícara de café, para café (36\%); unidade, para pão de sal (88\%) e bife, para carne bovina frita (54\%). Medidas equivocadas ou incompatíveis com os alimentos e unidades de medida que dificilmente podem ser dimensionadas foram citadas e uma unidade de medida padrão foi utilizada para estimar a quantidade consumida nessas situações.

\section{Conclusão}

Na estimativa das quantidades relatadas no Inquérito Nacional de Alimentação foram adotados procedimentos no intuito de minimizar os erros de mensuração. Esforços para melhorar a quantificação dos alimentos consumidos em inquéritos nacionais se justificam uma vez que a padronização das medidas referidas em inquéritos nutricionais possibilita comparações nacionais e internacionais e contribui para elaboração de recomendações e guias de alimentação e nutrição.

Termos de indexação: Coleta de dados. Consumo de alimentos. Registros de dieta.

\section{A B S T R A C T}

\section{Objective}

The present study described the methods used for estimating the weight and volume of the most consumed foods in Brazil reported in cooking units and presented suggestions that improve the assessment of food consumption reported in dietary surveys in Brazil.

\section{Methods}

Data from the National Dietary Survey of 2008-2009 were used for describing the rates of measurements reported for the five most commonly consumed items in Brazil (rice, beans, coffee, bread and beef steak), illustrating the construction of the database used in National Dietary Survey and discussing the main difficulties found in the process.

\section{Results}

Around 11,800 units of measurement associated with approximately 2,000 foods/preparations were cited. The serving spoon was the most cited measure for rice (57\%); ladle for beans (80\%); coffee cup for coffee (36\%); unit for bread (88\%), and unit for beef steak (54\%). Misleading and incompatible measurements and those that can hardly be estimated were cited and a standard unit of measurement was used for estimating the amount consumed in these situations.

\section{Conclusion}

Procedures were used for minimizing measurement errors when estimating quantities of foods consumed reported by the National Dietary Survey. Efforts to improve the quantification of food intake in national surveys are justified since the standardization of the measures reported in dietary surveys enables national and international comparisons and helps to develop recommendations and guidelines on food and nutrition.

Indexing terms: Data collection. Food consumption. Diet records.

\section{N T R O D U ÇÃ O}

Como todo procedimento de aferição, a avaliação do consumo alimentar está sujeita a erros que podem comprometer as análises de associação e de relação causal entre dieta e doença ${ }^{1}$. São diversas as fontes de erro nos inquéritos dietéticos, dentre as quais se destacam os problemas enfrentados na codificação e na estimativa das quantidades relatadas pelos participantes da investigação ${ }^{2-4}$. Rumpler et al. ${ }^{5}$ pesquisaram a magnitude e a contribuição relativa de diferentes fontes de erro de mensuração presente na estimativa do consumo alimentar e observaram que a estimativa das quantidades consumidas representou um terço do erro associado à ingestão de energia, carboidratos e gorduras, e metade do erro associada ao consumo de proteínas ${ }^{5}$.

Reportar a quantidade dos alimentos consumidos é uma tarefa complicada para os respondentes. Para alguns alimentos, as porções típicas ou naturais permitem um relato mais simplificado, por exemplo, ovo, pão francês ou pão 
de sal, banana, maçã etc. Porém, para alimentos relatados com base em unidades de medidas variadas - por exemplo, arroz, feijão, carne ou preparações mistas, como ensopado -, a estimativa da quantidade consumida requer procedimentos padronizados para construir uma base de dados consistente de conversão das quantidades e unidades de medidas citadas pelos entrevistados em medidas de massa e de volume.

Com o objetivo de conhecer o consumo alimentar da população brasileira, a Pesquisa de Orçamentos Familiares (POF) 2008-2009, desenvolvida pelo Instituto Brasileiro de Geografia e Estatística (IBGE), realizou o Inquérito Nacional de Alimentação (INA), módulo de avaliação do consumo alimentar individual por meio de registros alimentares, em uma subamostra de $25 \%$ dos domicílios investigados na POF.

O processo de elaboração da base de dados que permitiu estimar as quantidades consumidas a partir das unidades de medida referidas nos registros alimentares foi detalhado em publicação específica ${ }^{6}$. Este artigo tem como objetivos descrever os procedimentos empregados na preparação da base de dados das medidas caseiras referidas no Inquérito Nacional de Alimentação (INA) da POF 2008-2009, discutir as dificuldades enfrentadas na elaboração da referida base de dados, tomando como exemplo as medidas adotadas para os itens mais consumidos no Brasil, e apresentar propostas que possam ser adotadas para o aprimoramento do cálculo das quantidades ingeridas nos inquéritos alimentares no Brasil.

\section{M É T O D O S}

Os dados do presente artigo são de base secundária, provenientes do INA, módulo de consumo alimentar individual da POF 2008-2009. A POF 2008-2009 foi conduzida em uma amostra representativa de 55970 domicílios brasileiros. Os dados de consumo alimentar foram coletados em uma subamostra da POF 2008-2009, correspondente a $25 \%$ de todos os domicílios investigados na pesquisa. Nesses domicílios, foram obtidas informações sobre o consumo individual de alimentos de pelo menos um morador acima de 10 anos de idade, totalizando 34003 indivíduos.

O presente estudo foi aprovado pelo Comitê de Ética em Pesquisa do Instituto de Medicina Social da Universidade do Estado do Rio de Janeiro, Protocolo $n^{\circ}$ CAAE 0011.0259.000-11, em 19 de julho de 2011.

\section{Coleta de dados}

A coleta dos dados de consumo foi realizada por meio de registro alimentar em dois dias não consecutivos, visto que pelo menos dois dias de consumo são necessários para estimar consumo usual de alimentos ${ }^{7}$. O registro de alimentos compreendeu a informação detalhada dos alimentos consumidos, do tipo de preparação utilizada para alimentos específicos, como carnes e legumes, da porção e da quantidade consumidas, do horário, e do local de consumo. Todos os participantes do módulo de consumo alimentar receberam uma caderneta instrucional com explicações de como preencher o registro de alimentos e com fotos de utensílios e vasilhames que usualmente são utilizados para servir alimentos, por exemplo, diferentes tipos de pratos (de jantar, sobremesa, de sopa, pires), colheres e copos para auxiliar a estimativa das quantidades consumidas.

Os registros alimentares foram revisados e corrigidos pelos agentes de pesquisa, os quais armazenavam, ainda no próprio domicílio, as informações em programa computadorizado de entrada de dados desenvolvido pelo IBGE. O programa disponibilizava 15 opções de forma de preparação: crua, cozida, grelhada/brasa/churrasco, assada, frita, empanada/à milanesa, refogada, com molho vermelho, com molho branco, ao alho e óleo, com manteiga/óleo, ao vinagrete, ensopada, mingau e sopa. Para o relato da quantidade de alimento consumida, foram disponibilizadas 106 opções de unidades de medida (Quadro 1). 
648 | IN BEZERRA et al.

Quadro 1. Unidades de medidas utilizadas no Inquérito Nacional de alimentação da Pesquisa de Orçamentos Familiares $2008-2009$. Brasil, 2008-2009.

\begin{tabular}{|c|c|c|c|}
\hline Código & Medidas & Código & Medidas \\
\hline 1 & Asa & 54 & Garrafa (600mL) \\
\hline 2 & Bago & 55 & Garrafa (650mL) \\
\hline 3 & Banda & 56 & Garrafa (900mL) \\
\hline 4 & Barra & 57 & Garrafa (910mL) \\
\hline 5 & Bife & 58 & Garrafa (980mL) \\
\hline 6 & Bisnaga & 59 & Garrafa (1L) \\
\hline 7 & Bola & 60 & Garrafa (1,25L) \\
\hline 8 & Cacho & 61 & Garrafa $(1,5 \mathrm{~L})$ \\
\hline 9 & Caneca & 62 & Garrafa $(1,75 \mathrm{~L})$ \\
\hline 10 & Caneco & 63 & Garrafa (2L) \\
\hline 11 & Casquinha & 64 & Garrafa $(2,25 \mathrm{~L})$ \\
\hline 12 & Colher de arroz/servir & 65 & Garrafa $(2,5 \mathrm{~L})$ \\
\hline 13 & Colher de café & 66 & Garrafa (não especificada) \\
\hline 14 & Colher de chá & 67 & Gomo \\
\hline 15 & Colher de sobremesa & 68 & Grama \\
\hline 16 & Colher de sopa & 69 & Lata $(250 \mathrm{~mL})$ \\
\hline 17 & Concha & 70 & Lata $(335 \mathrm{~mL})$ \\
\hline 18 & Copo americano & 71 & Lata $(340 \mathrm{~mL})$ \\
\hline 19 & Copo de cafezinho & 72 & Lata $(350 \mathrm{~mL})$ \\
\hline 20 & Copo de requeijão & 73 & Lata (354mL) \\
\hline 21 & Copo grande & 74 & Lata $(473 \mathrm{~mL})$ \\
\hline 22 & Copo médio & 75 & Lata (não especificada) \\
\hline 23 & Copo tulipa & 76 & Litro \\
\hline 24 & Costela & 77 & Maço \\
\hline 25 & Coxa & 78 & Metade \\
\hline 26 & Cumbuca & 79 & Mililitro \\
\hline 27 & Dose & 80 & Pacote \\
\hline 28 & Escumadeira & 81 & Pedaço \\
\hline 29 & Espetinho & 82 & Pegador \\
\hline 30 & Espeto & 83 & Peito \\
\hline 31 & Espiga & 84 & Pescoço \\
\hline 32 & Fatia & 85 & Pires \\
\hline 33 & Filé & 86 & Ponta de faca \\
\hline 34 & Folha & 87 & Porção \\
\hline 35 & Garfada & 88 & Punhado \\
\hline 36 & Garrafa (200mL) & 89 & Posta \\
\hline 37 & Garrafa (237mL) & 90 & Pote \\
\hline 38 & Garrafa (248mL) & 91 & Prato de sobremesa \\
\hline 39 & Garrafa $(250 \mathrm{~mL})$ & 92 & Prato fundo \\
\hline 40 & Garrafa $(275 \mathrm{~mL})$ & 93 & Prato raso \\
\hline 41 & Garrafa (284mL) & 94 & Quilo \\
\hline 42 & Garrafa $(290 \mathrm{~mL})$ & 95 & Ramo \\
\hline 43 & Garrafa (300mL) & 96 & Rodela \\
\hline 44 & Garrafa $(320 \mathrm{~mL})$ & 97 & Sachê \\
\hline 45 & Garrafa (330mL) & 98 & Saco \\
\hline 46 & Garrafa $(350 \mathrm{~mL})$ & 99 & Sobrecoxa \\
\hline 47 & Garrafa (355mL) & 100 & Tablete \\
\hline 48 & Garrafa $(400 \mathrm{~mL})$ & 101 & Taca \\
\hline 49 & Garrafa $(450 \mathrm{~mL})$ & 102 & Tigela \\
\hline 50 & Garrafa $(500 \mathrm{~mL})$ & 103 & Unidade \\
\hline 51 & Garrafa $(510 \mathrm{~mL})$ & 104 & Unidade pequena \\
\hline 52 & Garrafa $(550 \mathrm{~mL})$ & 105 & Xícara de café \\
\hline 53 & Garrafa (591mL) & 106 & Xícara de chá \\
\hline
\end{tabular}




\section{Elaboração da base de dados das quantidades e das unidades de medida referidas para os alimentos citados no INA 2008-2009}

Uma descrição detalhada dos métodos utilizados na elaboração da base de dados das quantidades referidas para os alimentos consumidos no INA 2008-2009 constitui publicação específica dedicada somente a esse tópico ${ }^{6}$. Resumidamente, foram listadas, em ordem decrescente de citação, as unidades de medidas referidas para cada alimento, as quais foram associadas a medidas de massa e de volume a partir da compilação de tabelas de medidas caseiras, livros e manuais com informação sobre medidas caseiras de alimentos ${ }^{8-14}$; também foram utilizadas informações contidas em embalagens de alimentos e artigos científicos que especificavam o peso da unidade de frutas regionais ${ }^{15-17}$. Por fim, para alimentos e preparações relatados em unidades para os quais não se obteve informação sobre peso ou volume, nutricionistas treinadas desenvolveram pesagem em laboratório por meio de utensílios padronizados e de balança de precisão. O volume dos alimentos líquidos foi estimado em gramas: um mililitro foi considerado um grama, e um litro, um quilograma. Portanto, para cada alimento ou preparação citado nos registros, há um conjunto de unidades de medidas de massa em gramas. Para estimar a gramatura de todas as unidades de medida referidas no inquérito, foram adotados os procedimentos, em ordem de prioridade, conforme descrito na Figura 1.

Alguns alimentos preparados por diluição e consumidos na forma líquida foram relatados com unidades de medida na forma sólida do alimento, como consumo de café por meio de colher de café, colher de chá ou colher de sobremesa, indicando a quantidade de café em pó utilizada no preparo da bebida e não o consumo direto da bebida com esses utensílios. Todas essas medidas foram consideradas como sendo equivalentes a uma xícara de café $(50 \mathrm{~mL})$, ou seja, considerou-se que essas medidas foram utilizadas para o alimento em pó e que a quantidade em pó contida em uma colher de café ou chá ou sobremesa seria semelhante a uma xícara de café da bebida sob a forma líquida.

Situação semelhante foi observada entre alimentos sólidos que, após diluição, são consumidos na forma líquida, como o caso do leite em pó, achocolatado em pó, café solúvel em pó, entre outros. Alguns indivíduos reportaram o consumo de um copo médio de leite em pó, o que provavelmente refere-se ao consumo de um copo médio de leite líquido preparado a partir de leite em pó. Nesse caso, optou-se pela diluição do produto segundo a recomendação do rótulo da embalagem desse produto para o preparo da bebida; a mesma situação foi observada com o consumo de algumas frutas: por exemplo, um indivíduo relatou consumir um copo de graviola, o que muito provavelmente refere-se a um copo de suco de graviola. Nesses casos, inferiu-se que o consumo era referente ao suco da fruta e, portanto, considerou-se

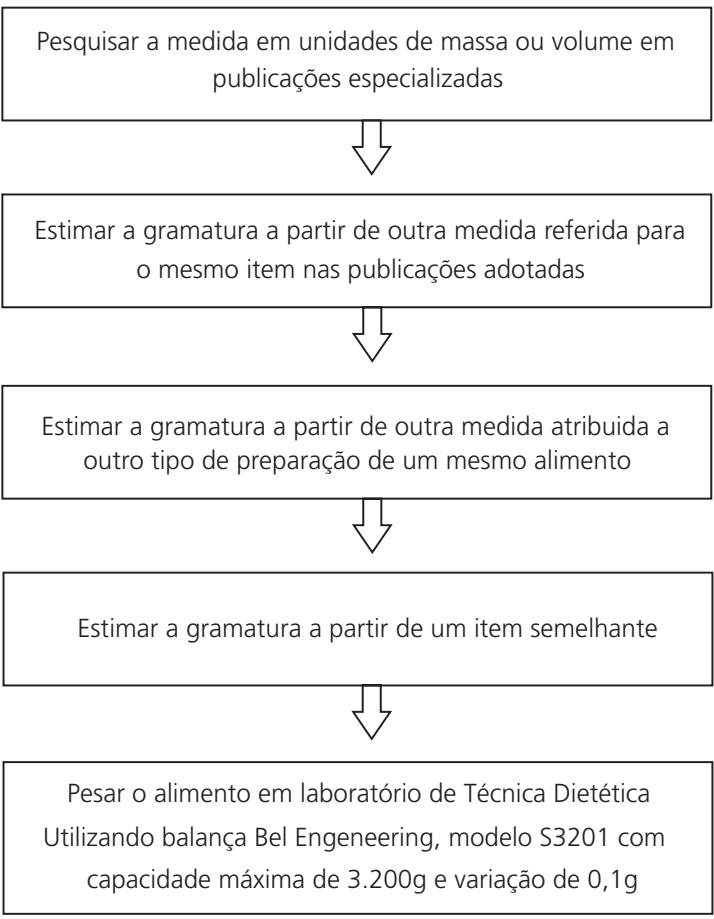

Figura 1. Procedimentos utilizados para estimar medidas de massa e de volume dos alimentos consumidos no Inquérito Nacional de Alimentação da Pesquisa de Orçamentos Familiares 2008-2009. Brasil, 2008-2009. 
a diluição de $100 \mathrm{~g}$ da fruta para o preparo de um copo médio de $240 \mathrm{~mL}$.

Quando não foi possível adotar nenhum dos procedimentos descritos anteriormente para estimar a gramatura da medida citada, estabeleceu-se que a unidade de medida citada com maior frequência para o alimento específico seria considerada "unidade de medida padrão", tendo sido definida uma unidade de medida padrão para cada alimento.

\section{Análise dos dados}

Foram identificados os cinco itens mais citados no inquérito dietético a partir da estimativa da frequência de todos os alimentos citados nos dois dias de registro alimentar para ilustrar a construção da base de dados de medidas caseiras utilizada na análise dos dados de consumo alimentar individual do INA 2008-2009. Em seguida, as frequências brutas das unidades de medida referidas para esses itens foram descritas.

\section{RES U LTA DOS}

Nos registros alimentares obtidos no INA 2008-2009, foram referidos aproximadamente 2000 alimentos ou preparações, aos quais foram relacionadas cerca de 11800 quantidades em diferentes unidades de medidas, para as quais se estimou a quantidade em medidas de peso ou volume. Três por cento dessas unidades de medida foram estimadas a partir de pesagem dos alimentos e de preparações; em $6 \%$ das informações foram utilizadas as medidas propostas no Guia Alimentar para a População Brasileira18, e para $8 \%$ das informações, foram utilizadas outras fontes bibliográficas ${ }^{8-11,13-17}$. Para $40 \%$ dessas unidades de medida, a quantidade em gramas era previamente conhecida, pois se referiam a embalagens ou a garrafas e latas com pesos e volumes pré-definidos. Outros $43 \%$ desses itens foram compilados a partir da Tabela para Avaliação do Consumo Alimentar em Medidas Caseiras ${ }^{12}$.
Com base nos dois dias de consumo, os cinco alimentos mais citados no Brasil foram: arroz, feijão, pão de sal, café e carne bovina frita. Para referir o consumo desses alimentos, foram utilizadas 55 unidades de medida diferentes, constituindo um universo de 253080 citações. $O$ arroz foi referido por $90 \%$ dos respondentes. Para esse item, foram utilizadas 28 unidades de medida em 80033 citações. O feijão foi consumido por $80 \%$ dos respondentes e foi associado a 27 unidades de medida, totalizando 62662 citações. O pão foi citado 39152 vezes, tendo sido referido por $57 \%$ dos indivíduos entrevistados em 20 unidades de medida. Para descrever o consumo de café, $63 \%$ dos respondentes do inquérito adotaram 34 unidades de medida, sendo registradas 56932 citações para esse item. A carne bovina frita foi consumida por $28 \%$ dos entrevistados, com o uso de 23 unidades de medida, totalizando 14301 citações.

Em relação à frequência de citação das medidas referidas para arroz, feijão, café, pão de sal e carne bovina frita, a colher de servir foi a medida mais citada para o arroz (57\%); a concha, para o feijão (80\%); a xícara de café, para o café $(36 \%)$; a unidade, para pão de sal (88\%), e bife, para carne bovina frita (54\%) (Figura 2). Pode-se observar que o relato do consumo desses alimentos em gramas, quilo, litro ou mililitro foi pouco frequente (menos de 1,5\% das citações), e que a unidade de medida "porção" também foi citada com frequência reduzida (variando de 0,01\% para pão e café a $4 \%$ para arroz). Em todo o inquérito, $5 \%$ dos alimentos registrados foram citados com a medida "porção".

Algumas unidades de medidas referidas para esses alimentos foram consideradas inusitadas: "colher de café" utilizada para o consumo de feijão e "concha" para café. Apesar da baixa frequência de citação dessas unidades de medida, elas constam da base de dados, pois, uma vez registradas, era necessário atribuir-lhes alguma medida de massa ou volume.

Observou-se o relato de unidades de medidas que foram possivelmente registradas de forma 

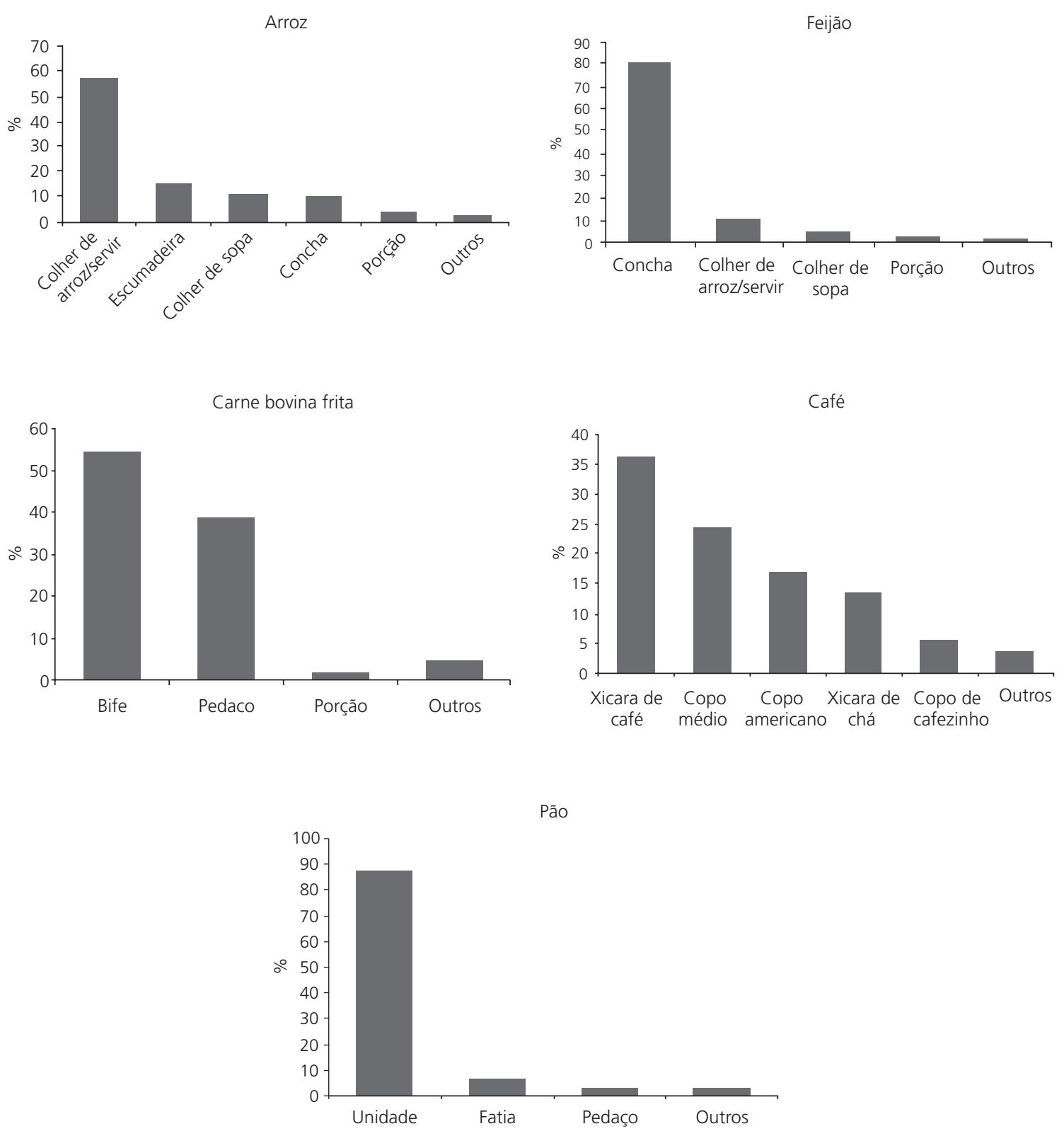

Figura 2. Frequência (\%) de citação das unidades de medidas adotadas para referir o consumo de alimentos selecionados no Inquérito Nacional de Alimentação da Pesquisa de Orçamentos Familiares 2008-2009. Brasil, 2008-2009.

equivocada e eram incompatíveis aos alimentos: coxa e sobrecoxa de café $(0,01 \%$ das unidades de medida atribuídas a esse item) e pedaço ou banda de feijão $(0,004 \%$ das unidades de medida desse item). Também observou-se a ocorrência de unidades de medida que dificilmente podem ser dimensionadas, como unidade de arroz e punhado de carne bovina $(0,03 \%$ das medidas citadas para o arroz e para a carne bovina frita).
Nesses casos, adotou-se o procedimento de imputar o valor de uma unidade de medida considerada padrão para cada alimento específico. Para aproximadamente 3\% das unidades de medida citadas nos registros alimentares, foi preciso imputar a quantidade a partir da medida padrão.

No caso do arroz, a medida padrão foi a colher de servir; para as unidades de medida 
pedaço, pescoço, punhado e unidade, foi atribuída a gramatura da "colher de servir" de arroz (45g), que correspondeu a $0,05 \%$ de todos os registros observados para o arroz. No caso do feijão, às unidades de medida banda, coxa, metade, pedaço, unidade e unidade pequena foi atribuída a gramatura da "concha" (140g), que foi a unidade de medida padrão, o que correspondeu a $0,03 \%$ de todos os registros observados para feijão. Para o café, a xícara de café ( $50 \mathrm{ml})$ foi considerada a unidade de medida padrão para 0,2\% das medidas citadas para esse item (cacho, diferentes tipos de colher, concha, coxa e sobrecoxa, metade, pedaço, ponta de faca e unidade). Para o pão de sal, atribuiu-se a gramatura de "uma unidade" (50g) como medida padrão para 0,08\% dos registros referentes às unidades de medida concha, copo americano, copo grande, copo médio, escumadeira, ponta de faca, punhado, prato fundo, tigela e xícara de café. No caso da carne bovina frita, foi atribuída às unidades de medida metade e punhado a unidade de medida "bife" (100g), considerada a unidade de medida padrão para esse alimento, o que correspondeu a 0,04\% das medidas citadas para esse item.

\section{I S C U S S Ã O}

Este estudo apresenta os procedimentos empregados para o dimensionamento das quantidades relatadas para descrever o consumo dos alimentos mais consumidos no Brasil (arroz, feijão, café, pão e carne bovina frita) com base nos dados do Inquérito Nacional de Alimentação da POF 2008-2009. A base de dados de medidas caseiras utilizada na análise dos dados desse importante inquérito de amplitude nacional e de base populacional é resultado da compilação de dados de informações contidas em literatura especializada, e foi publicada pelo IBGE, em junho de 2011, na "Tabela de medidas referidas para os alimentos consumidos no Brasil" ${ }^{6}$. Nessa tabela, são apresentadas as estimativas em gramas para a maioria das medidas citadas e são descritos os proce- dimentos adotados para a imputação da gramatura de unidades de medida equivocadas ou incompatíveis com os alimentos aos quais estavam relacionadas e também aquelas unidades de medida que dificilmente podem ser dimensionadas.

Registros equivocados referem-se provavelmente a erro na entrada dos dados, como, por exemplo, "sobrecoxa de café". Unidades de medida incompatíveis com determinados alimentos podem ser exemplificadas por registros como "pedaço de feijão". Para as unidades de medidas inusitadas, apesar de compatíveis com os alimentos a que foram associados, não se dispunha de suas quantidades em gramas nas publicações de referência, como, por exemlo, "colher de café de feijão".

O uso de unidades de medidas inapropriadas para determinados alimentos pode apontar a dificuldade dos indivíduos em estimar a quantidade consumida ou pode simplesmente ser efeito de erro de digitação na entrada de dados. O uso da unidade de medida 'porção' pode também indicar dificuldade do respondente para estimar a quantidade consumida do alimento, o que o faz optar por uma descrição mais geral. No inquérito alimentar da POF 2008-2009, 'porção' foi reportada em menos de $5 \%$ das citações, possivelmente porque o programa para entrada de dados disponibilizava 106 diferentes unidades de medida e devido ao uso de fotografias de vasilhames e talheres que foram distribuídas aos respondentes a fim de auxiliar-Ihes o relato das quantidades consumidas.

A referência a unidades de medida pouco usuais ou que não fazem sentido para relatar o consumo de determinado item, por exemplo, o uso da colher de café para relatar o consumo de arroz, pode refletir erro do entrevistado ou falta de crítica por parte do entrevistador no momento da entrada de dados. A adequada capacitação dos entrevistadores de inquéritos dietéticos é extremamente necessária para que a revisão dos registros e a inclusão dos dados reportados pelos entrevistados ocorram de forma apropriada e com o devido discernimento sobre a adequação da uni- 
dade de medida ao tipo de alimento ${ }^{1}$. A restrição das unidades de medida de acordo com o tipo de alimento poderia ajudar a reduzir esse tipo de erro.

Na hipótese de que utensílios não usuais tenham sido usados para servir determinados alimentos, pode-se considerar que essa situação é indício de um problema ainda maior e não mensurável pelo inquérito, que é a falta de utensílios adequados para servir alimentos em alguns domicílios brasileiros. Alguns utensílios, apesar de não usuais, são possíveis de serem utilizados para servir ou consumir alimentos. Nessas situações, a pesagem de alimentos foi realizada para alguns itens citados no inquérito. Esse procedimento, apesar de ser uma solução rápida e direta, também incorpora problemas importantes, como a dificuldade na padronização dos utensílios correspondentes às porções indicadas pelos entrevistados e a limitação em se obterem alimentos típicos de determinadas regiões do Brasil ou disponíveis apenas em períodos restritos do ano, como, por exemplo, coco mucajá, palma, entre outros.

A estimativa da quantidade dos alimentos consumidos constitui uma importante fonte de erro em inquéritos dietéticos, e esse é o primeiro estudo a discutir os procedimentos utilizados na estimativa da quantidade de alimentos consumidos com base em um inquérito nacional de alimentação, o que preenche uma importante lacuna na literatura e contribui para a realização de futuros inquéritos dietéticos no País.

É importante destacar que inquéritos alimentares estão sujeitos a erros que podem ser aleatórios ou sistemáticos. Erros aleatórios inflam a variabilidade das distribuições de consumo, o que reduz o poder estatístico para se detectar alguma associação entre consumo de alimentos e desenvolvimento de doença e atenua e subestima as estimativas de possíveis associações ${ }^{1,7}$.

Erros sistemáticos como sub-relato de alimentos consumidos são mais sérios porque podem alterar os resultados ${ }^{1}$. A estimativa de massa e volume dos alimentos consumidos a partir de tabelas de medidas caseiras pode gerar um erro de natureza sistemática com a inclusão de gramaturas inapropriadas para as medidas citadas. Os procedimentos utilizados no INA tentaram minimizar a possibilidade desse tipo de erro por meio do uso de referências bibliográficas reconhecidas no País. Além disso, a imputação das quantidades padrões para medidas inusitadas, inapropriadas ou incompatíveis com o alimento ocorreu em proporções desprezíveis, o que não gera alterações significativas na média total de consumo da população.

O inquérito alimentar da POF 2008-2009 procurou minimizar uma importante fonte de erro aleatório, que é a variabilidade intrapessoal do consumo de alimentos, com o uso de dois registros alimentares, o que permite obter estimativas não enviesadas do consumo usual de alimentos ${ }^{19}$. $\mathrm{O}$ método do registro, que é considerado um método prospectivo, pois prevê o registro do consumo no momento em que o alimento é consumido, é tido como um dos mais acurados para a obtenção do tamanho das porções consumidas ${ }^{19}$. Além disso, reduziu-se a possibilidade de erro sistemático com a detalhada avaliação das medidas de massa e de volume citadas nos dois dias de inquérito. Dessa forma, foi possível incorporar todas as medidas de massa e de volume para todos os itens citados na pesquisa e, com isso, estimar a quantidade consumida pelos entrevistados.

No módulo de consumo da POF 2008-2009, 106 medidas de massa ou de volume foram disponíveis para estimativa da quantidade consumida pelos entrevistados. Apesar da baixa frequência de citação das medidas inusitadas, inapropriadas ou incompatíveis com os alimentos, esta análise demonstrou que a utilização de um conjunto único e ampliado de unidades de medida em inquéritos alimentares pode dar origem a relatos equivocados das quantidades consumidas pela seleção inadequada de opções. Nesse primeiro inquérito de consumo individual de alimentos conduzido em 2008-2009 em amostra probabilística representativa da população brasileira de todas as unidades da federação, todas as medidas de massa 
e de volume foram disponíveis para todos os itens alimentares de forma indiscriminada. A partir dessa primeira experiência, será possível melhorar a coleta de dados levando-se em conta reconhecimento das medidas mais frequentemente referidas para cada alimento e realizar modificações no programa de entrada de dados de modo a restringir as unidades de medidas para cada item, o que reduz a citação de medidas equivocadas, inapropriadas ou inusitadas, mesmo que nesse primeiro inquérito essas opções tenham aparecido de forma pouco importante. Esse procedimento facilita a elaboração do manual e o treinamento dos entrevistadores e também favorece o relato correto da quantidade consumida por parte do respondente.

Uma unidade de medida padrão foi estimada para cada alimento, e a utilização dessa medida nas situações em que não foi possível adotar nenhum dos procedimentos descritos para estimar a gramatura da medida citada faz parte do processo de crítica e controle de qualidade de dados provenientes de grandes inquéritos ${ }^{20}$. No inquérito dietético What We Eat In America (WWEIA), módulo de consumo do National Health and Nutrition Examination Survey (NHANES), realizado continuamente a cada dois anos nos Estados Unidos, uma medida padrão também é utilizada para estimar quantidades desconhecidas de alimentos ${ }^{21}$. No inquérito americano, a medida padrão é a mesma para todos os alimentos e corresponde a 1 cup, que equivale a aproximadamente $240 \mathrm{~mL}$ de água ou 175 gramas de arroz cozido. No inquérito brasileiro, a estimativa da medida padrão foi realizada para cada alimento separadamente, com base na medida citada com maior frequência para aquele alimento. Com esse procedimento, acredita-se obter um valor mais fidedigno da média populacional.

Com base em inquéritos dietéticos desenvolvidos em outros países e nas análises aqui apresentadas, fica evidente que a estimativa de massa e de volume das medidas caseiras de inquéritos dietéticos deve passar por revisão contínua. Vale salientar que o banco de dados com as informa- ções sobre os alimentos e medidas caseiras deve ser constantemente atualizado devido ao surgimento de novos alimentos, bem como de novas medidas e embalagens comerciais de produtos industrializados. É necessário que se formem grupos de pesquisa voltados para a revisão da tabela de medidas caseiras do INA e que surjam novas publicações com informações que alimentem a base de dados do IBGE. O ideal seria conduzir um plano regular de revisão, desenvolvido por categoria de alimentos com base na frequência de consumo da POF de 2008-2009.

Os procedimentos utilizados no INA 2008-2009 tentaram minimizar os vieses associados às estimativas das quantidades consumidas em inquéritos dietéticos, o que é considerado uma relevante fonte de erro nesses inquéritos. Limitar o número de medidas disponíveis por alimento, intensificar o treinamento dos entrevistadores de campo e estabelecer revisões regulares do banco de dados com informação sobre os alimentos e medidas caseiras são propostas que se justificam para melhor quantificar os alimentos consumidos em inquéritos nacionais. A padronização das medidas referidas em inquéritos nutricionais por meio da utilização do peso em gramas dos alimentos e sua estimativa em medidas de massa e de volume possibilitam comparações nacionais e internacionais e contribuem para elaboração de recomendações e guias de alimentação e nutrição.

\section{OLABORADORES}

IN BEZERRA e MC ARAUJO contribuíram com a compilação de dados para a elaboração da tabela de medidas referidas no Inquérito Nacional de Alimentação 2008-2009. LS MONTEIRO contribuiu com a pesagem de alimentos. IN BEZERRA, LS MONTEIRO e MC ARAUJO contribuíram com análise e interpretação dos dados. RA PEREIRA, EM YOKOO e R SICHIERI contribuíram com concepção, desenho, análise e interpretação dos dados. Todos os autores contribuíram substancialmente na elaboração do manuscrito ou na revisão crítica do conteúdo e aprovaram a versão final do manuscrito. 


\section{REFERÊ N CIAS}

1. Willett WC. Nutritional Epidemiology. $2^{\text {nd }}$ ed. New York: Oxford University Press; 1998.

2. Cypel YS, Guenther PM, Petot GJ. Validity of portion-size measurement AIDS: a review. J Am Diet Assoc. 1997; 97(3):289-92. doi: 10.1016/S0002-82 23(97)00074-6.

3. Lansky D, Brownell KD. Estimates of food quantity and calories: errors in self-report among obese patients. Am J Clin Nutr. 1982; 35(3):727-32.

4. Harnack L, Steffen L, Arnett DK, Gao S, Luepker RV. Accuracy of estimation of large food portions. J Am Diet Assoc. 2004; 104(5):804-6. doi: 10.1016/j. jada.2004.02.026\$0002822304002329 [pii].

5. Rumpler WV, Kramer M, Rhodes DG, Moshfegh AJ, Paul DR. Identifying sources of reporting error using measured food intake. Eur J Clin Nutr. 2008; 62(4):544-52. doi: 10.1038/sj.ejcn.1602742.

6. Instituto Brasileiro de Geografia e Estatística. Pesquisa de Orçamentos Familiares 2008-2009: tabela de medidas referidas para os alimentos consumidos no Brasil. Rio de Janeiro. IBGE; 2011.

7. Dodd KW, Guenther PM, Freedman LS, Subar AF,

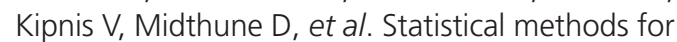
estimating usual intake of nutrients and foods: a review of the theory. J Am Diet Assoc. 2006; 106(10):1640-50. doi: 10.1016/j.jada.2006.07.011.

8. Fisberg RM, Villar BS. Manual de receitas e medidas caseiras para cálculo de inquéritos alimentares. Manual elaborado para auxiliar o processamento de dados de inquéritos alimentares. São Paulo: Signus; 2002.

9. Naves MMV, Silva MR, Silva MS, Oliveira AG. Culinária Goiana: valor nutritivo de pratos tradicionais. Goiânia: Kelps; 2004.

10. Pacheco M. Tabela de medidas caseiras e composição química dos alimentos. Rio de Janeiro: Rubio; 2006.

11. Philippi ST. Pirâmide dos alimentos: fundamentos básicos da nutrição. São Paulo: Manole; 2008.

12. Pinheiro ABV, Lacerda EMA, Benzecry EH, Gomes MCS, Costa VM. Tabela para avaliação de consumo alimentar em medidas caseiras. $5^{a}$ ed. São Paulo: Atheneu; 2001.

13. Tomita LY, Cardoso MA. Relação de medidas caseiras, composição química e receitas de alimentos nipo-brasileiros. São José do Rio Preto: Farmep; 2000.

14. Tuma R, Monteiro R. Tabela de alimentos equivalente. Belém: Nutrivisa; 1999.

15. Araújo RR, Santos ED, Lemos EEP, Alves RE. Caracterização biométrica de frutos e sementes de genótipos de murici (Byrsonima verbascifolia (L.) Rich.) do tabuleiro costeleiro de Alagoas. Revista Caatinga. 2009; 22(3):224-8.

16. Caldeira SD, Hiane PA, Ramos MIL, Ramos Filho MM. Caracterização físico-química do araçá (Psidium guineense sw.) e do tarumã (Vitex cymosa bert.) do Estado de Mato Grosso do Sul. Bol Centro Pesqui Process Aliment. 2004; 22(1):145-54.

17. Souza FG, Figueiredo RW, Alves RE, Maias GA, Araújo IA. Qualidade pós-colheita de frutos de diferentes clones de mangabeira (hancornia speciosa gomes). Ciênc Agrotec. 2007; 31(5):1449-54.

18. Brasil. Ministério da Saúde. Secretária de Atenção à Saúde. Departamento de atenção básica. Coordenação-Geral da Política de Alimentação e Nutrição. Guia alimentar para a população brasileira: promovendo a alimentação saudável. Brasil: MS; 2006.

19. Thompson FE, Subar AF. Dietary assessment methodology. In: Coulston AM, Boushey CJ, editors. Nutrition in the prevention and treatment of disease. $2^{\text {nd }}$ ed. London: Elsevier Academic Press; 2008.

20. Biemer PP, Lyberg LE. Data processing: errors and their control. introduction to survey quality. New Jersey: Wiley; 2003.

21. Anand J, Raper NR, Tong A. Quality assurance during data processing of food and nutrient intakes. J Food Compost Anal. 2006; 19:S86-S90. doi: 10.1016/j.jfca.2006.02.005.

Recebido em: 17/8/2011

Versão final em: 27/3/2012

Aprovado em: 25/6/2012 
